\title{
EVALUATION OF IL-6 AND HIGHY SENSITIVE C REACTIVE PROTEIN VALUE IN CSF AND SERUM CHILDREN WHO SUSPECTED MENINGITIS REFERRED TO PEDIATRIC EMERGENCY ROOM
}

\author{
A. Hamedi ${ }^{1}$, H. Ayatullahi ${ }^{2}$ \\ ${ }^{1}$ Pediatrics, ${ }^{2}$ Pathology, Mashhad University of Medical Science, Mashhad, Iran
}

Introduction: Acute bacterial meningitis which is a pediatric emergency must be diagnosed and treat promptly. Often diagnosis of Bacterial meningitis from viral meningitis is difficult after some days.

Determination of some inflammatory mediators example IL-6 and hsCRP were useful in differential diagnosis of bacterial and viral meningitis.

Objectives: Determination of hs CRP and IL6 in serum and CSF in children suspected meningitis.

Materials \& methods: From of children that hospitalized in pediatric emergency ward and for they perfomed lumbar puncture, $1 \mathrm{cc}$ serum and csf of they were taken to libratory ward and have measured IL-6 with Elisa method and hsCRP with Immunoturbidometry method .

Results: From 81 cases, 27 cases (33/3\%) were bacterial meningitis 27 cases (33/3\%) were viral meningitis and 27 cases $(33 / 3 \%)$ were normal. IL- 6 concentration in the csf and serum were significantly raised in cases of bacterial meningitis $(\mathrm{P}=0 / 001, \mathrm{P}=0 / 01)$ but hsCRP concentration in the CSF and serum not significantly raised in cases of bacterial meningitis . Mean IL-6 in serum in bacterial meningitis was (50.01) and in viral meningitis was (10.64) mean IL-6 concentration in the CSF in bacterial meningitis was (180.74) and in viral meningitis was (39.08) mean hsCRP in CSF in bacterial meningitis was (2.22) and viral meningitis was (1.29) mean hsCRP in serum in bacterial meningitis was (8.23) and viral meningitis was (6.36).

Conclusion: The measurement of IL-6 in the CSF and serum is potentially a very useful diagnostic tool for differential diagnosis of bacterial and viral meningitis. 